\title{
Percutaneous Transhepatic Biliary Interventions
}

\author{
Ankusha Yadav ${ }^{1}$ Naveen Kumar Condati ${ }^{1} \quad$ Amar Mukund ${ }^{1}$
}

${ }^{1}$ Department of Interventional Radiology, Institute of Liver and Biliary Sciences, New Delhi, India

J Clin Interv Radiol ISVIR 2018;2:27-37

\begin{abstract}
Address for correspondence Amar Mukund, MD, Department of Interventional Radiology, Institute of Liver and Biliary Sciences, Vascular Lab, Phase I, D1 Vasant Kunj, New Delhi 110070, India (e-mail: dramarmukund@gmail.com).
\end{abstract}

Abstract

Keywords
- biliary interventions
- percutaneous transhe-
patic biliary drainage
- biliary obstruction

Biliary tract interventions remain indispensable procedures for treatment of a wide arena of biliary tract pathologies. The increased use of endoscopic retrograde cholangiopancreatography (ERCP) for biliary tract evaluation and intervention has resulted in fewer patients requiring percutaneous transhepatic biliary interventions. However, there remains a subset of patients in whom ERCP is not feasible. Interventional radiologists play an important role in the management of biliary pathologies in such patients who cannot be benefited by endoscopic means. These interventions may at times be challenging; hence, awareness of normal and variant biliary tract anatomy and the variety of biliary tract interventions make the job easy. This article reviews the role of percutaneous transhepatic interventions in the management of a variety of biliary tract diseases, both benign and malignant, general indications and contraindications, preprocedural evaluation, and technical considerations specific to indications.

\section{Introduction}

Percutaneous biliary interventions are used in a multitude of benign and malignant conditions. The basic biliary interventions are percutaneous transhepatic cholangiography (PTC), percutaneous cholecystostomy (PCC), and percutaneous transhepatic biliary drainage (PTBD). The origin of percutaneous biliary interventions dates back to around 1921 when direct puncture of the gallbladder (GB) was first performed to opacify the biliary tree. The technique of PTBD for obstructive biliopathies was introduced in the 1970s and PCC in the 1980s. ${ }^{1-4}$ Development of biliary stents further widened the scope of percutaneous biliary interventions.

Nowadays, numerous other novel applications of PTBD have been developed, which will be further discussed at appropriate places. Careful patient selection, review of preprocedural diagnostic imaging studies, appropriate planning, and availability of support personnel are necessary prerequisites while planning a percutaneous biliary intervention. ${ }^{5}$

\section{General Indications to Percutaneous Biliary Interventions}

Impaired biliary drainage may occur due to benign or malignant obstruction that leads to hyperbilirubinemia, cholangitis, pruritus, and other constitutional symptoms. Percutaneous biliary drainage is usually required to relieve obstruction when endoscopic biliary drainage cannot be performed. ${ }^{6}$

Benign causes of biliary obstruction can be iatrogenic in nature, and most commonly resulting from injury to the biliary tree during surgical procedures, particularly laparoscopic cholecystectomy, biliary-enteric anastomoses, and liver transplantation. Also, benign biliary obstruction can be associated with inflammatory processes such as chronic pancreatitis, sclerosing or recurrent pyogenic cholangitis, and sphincter of Oddi's dysfunction or papillary stenosis. ${ }^{78}$ Hepatic arterial chemotherapeutic infusions and radiotherapy for upper abdominal malignancies can also induce benign biliary strictures that may require PTBD. PTBD may be indicated in some cases of portal biliopathy in which in the biliary dilation is caused by both mechanical obstruction and compressive ischemia of the biliary tract. Mirizzi's syndrome is another rare indication, in which in the impacted gallstone can induce repeated inflammation and subsequent stricture formation. ${ }^{9}$

Few other common indications for which biliary intervention are done include postsurgical biliary leak, biliary calculi, and abdominal blunt or penetrating trauma. Malignant biliary duct obstruction can be associated with carcinoma of the $\mathrm{GB}$, periampullary tumor, cholangiocarcinoma, and metastatic disease to periportal lymph nodes ( - Tables $\mathbf{1}, \mathbf{2}$ ). ${ }^{10}$
DOI https://doi.org/

10.1055/s-0038-1642105.

ISSN 2457-0214.
Copyright @2018 by Indian Society of Vascular and Interventional Radiology
License terms

(요 (1) $\odot \circledast$ 
Table 1 Indications for PTBD in benign biliary obstruction

\begin{tabular}{|c|c|}
\hline \multirow[t]{3}{*}{ Postoperative strictures } & - latrogenic injury following laparoscopic cholecystectomy \\
\hline & - Biliary-enteric anastomoses \\
\hline & - Liver transplantation \\
\hline \multirow[t]{4}{*}{ Inflammatory processes } & - Chronic pancreatitis \\
\hline & - Sclerosing cholangitis \\
\hline & - Recurrent pyogenic cholangitis \\
\hline & - Mirizzi’s syndrome. \\
\hline \multirow[t]{2}{*}{ Mechanical obstruction } & - Portal biliopathy \\
\hline & - Biliary calculi \\
\hline \multirow[t]{5}{*}{ Miscellaneous } & - Postsurgical biliary leaks \\
\hline & - Sphincter of Oddi's dysfunction or papillary stenosis \\
\hline & - Abdominal trauma, blunt or penetrating \\
\hline & - Radiotherapy to upper abdomen \\
\hline & - Chemotherapy-hepatic arterial continuous infusions \\
\hline
\end{tabular}

Abbreviation: PTBD, percutaneous transhepatic biliary drainage.

Table 2 Indications for PTBD in malignant biliary obstruction

\begin{tabular}{|l|}
\hline - Gallbladder carcinoma \\
\hline - Periampullary carcinoma \\
\hline - Cholangiocarcinoma \\
\hline - Pancreatic carcinoma \\
\hline $\begin{array}{l}\text { lymph nodes } \\
\text { l Metastasis }\end{array}$ \\
\hline
\end{tabular}

Abbreviation: PTBD, percutaneous transhepatic biliary drainage.

\section{General Contraindications to Percutaneous Biliary Interventions}

There are no absolute contraindications for percutaneous biliary interventions, though there are several relative contraindications that include the following ${ }^{11}$ :

- Coagulopathy: Correction of the coagulopathy to an international normalized ratio (INR) of 1.5 or less and platelet transfusion to at least 50,000 is recommended.

- Perihepatic fluid/ascites can cause pericatheter leakage of bile and may lead to biliary peritonitis. Preprocedural paracentesis should be considered in such cases. Also, the left-sided biliary access is preferable as it provides a route that is away from ascites.

\section{Preprocedural Evaluation and Preparation}

All patients with obstructive biliopathy should undergo detailed clinical evaluation and relevant laboratory tests. Imaging studies such as ultrasonography, multiphase contrast-enhanced computed tomography (CT) scan, and/or magnetic resonance imaging (MRI) with magnetic resonance cholangiopancreatography (MRCP) prior to percutaneous interventions are useful in identifying the level and cause of obstruction and also in defining the extent of the disease, thus providing a platform to plan further management. ${ }^{12}$ Also, the presence of any variations in biliary anatomy ( $\mathbf{- F i g . ~ 1})^{13}$ can be identified. Coagulation parameters should be checked, which routinely include prothrombin time (PT), INR, and platelet count. Broad-spectrum antibiotics that provide coverage for both gram-positive and -negative organisms are routinely given to the patients prior to the procedure as transient bacteremia occurs during the procedure. ${ }^{14}$

Third-generation cephalosporins (injection ceftriaxone or cefixime $1 \mathrm{~g}$ intravenously [IV]) can be routinely administered 1 hour prior to the procedure. Piperacillin-tazobactam combination (4.5 g IV) is the preferable preprocedural antibiotic in patients presenting with biliary sepsis. ${ }^{15}$

The procedure is generally performed under local anesthesia (2\% lidocaine), and preprocedural analgesia is provided with IV paracetamol $1 \mathrm{~g}$ infusion and a loading dose of fentanyl $50 \mu \mathrm{g}$ IV, followed by top-up doses of fentanyl 25 to $50 \mu \mathrm{g}$ IV as required. However, in some cases, conscious sedation is required to provide adequate pain relief and elective procedures can be taken up after following the institute's guidelines for sedation. ${ }^{16-18}$ The American Society of Anesthesiologists usually recommend 2 hours of fasting for clear fluids and 6 hours of fasting for a light meal prior to sedation/analgesia. ${ }^{16}$

General anesthesia in percutaneous transhepatic biliary interventions may be required in pediatric age group, noncooperative anxious patients, and also when the procedure time is expected to be long in complex and difficult cases. ${ }^{18}$

\section{Percutaneous Transhepatic Cholangiography}

Percutaneous transhepatic cholangiography (PTC) is a procedure that involves placement of a fine needle into a peripheral biliary radicle under image guidance followed by contrast injection to opacify the biliary tree. PTC was a commonly performed procedure in the 1980s and the early 1990s. Subsequently, ERCP became an increasingly popular modality for both the diagnosis and the treatment of biliary tract disorders. Then, the emergence of MRCP technique that is noninvasive 

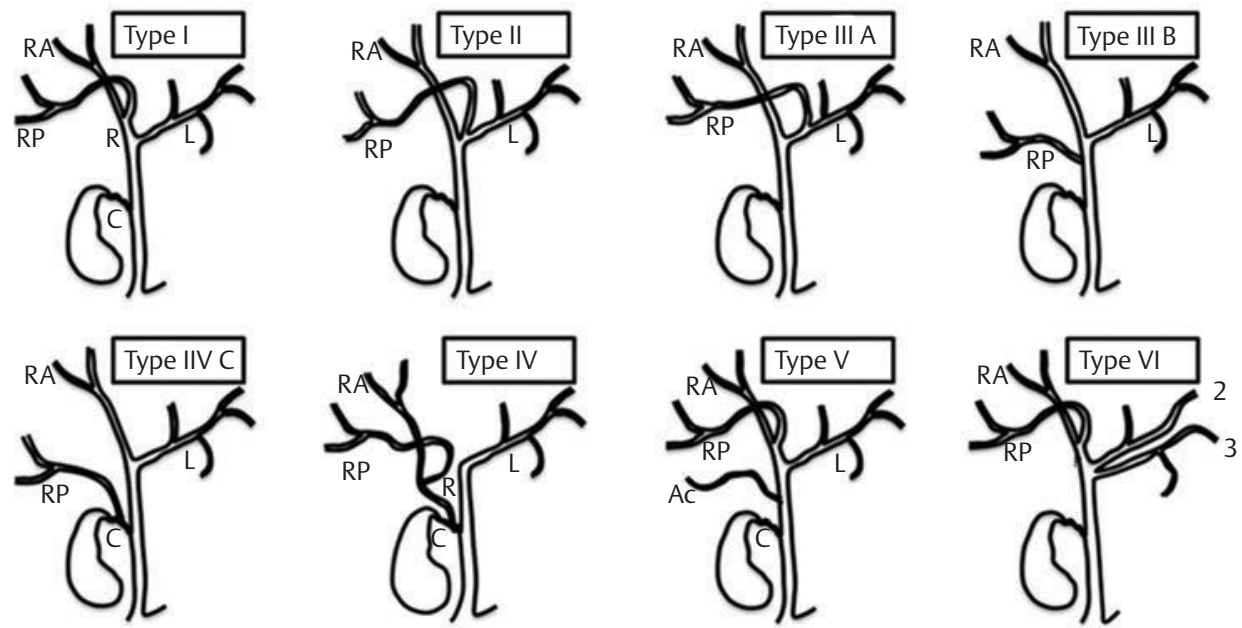

Fig. 1 Pictorial diagram showing variations in the right biliary ductal system. Type I is the most common and normal variant. Ac, accessory duct; C, cystic duct; L, left hepatic duct; R, right hepatic duct; RA, right anterior duct; RP, right posterior duct.

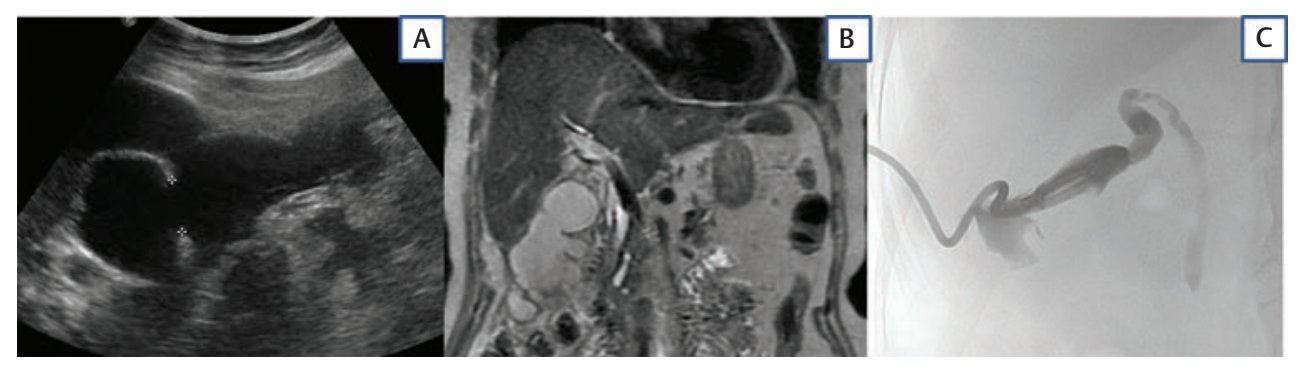

Fig. 2 Percutaneous cholecystotomy (PCC). A case of perforated gallbladder demonstrating a rent on ultrasound and MR studies (A, B). Fluoroscopic image $(\mathrm{C})$ demonstrating an $8 \mathrm{~F}$ pigtail catheter percutaneously inserted into the gallbladder body.

and free of contrast and radiation-related risks limited the sole diagnostic use of PTC and ERCP. Currently, the role of PTC is confined to those conditions in which access to the biliary system for therapeutic interventions is also desired. ${ }^{14,19}$

\section{Percutaneous Cholecystotomy}

This minimally invasive image-guided therapy is used in the management of acute cholecystitis in patients who are not good surgical candidates or as a bridge to definitive surgery. ${ }^{20,21}$ The main goal in this patient population is drainage of the GB and prevention of impending perforation and generalized sepsis. It can also be used to gain access to the biliary system for doing further procedures such as stone extraction, lithotripsy when endoscopic or transhepatic routes are difficult or contraindicated..$^{22}$ Relative contraindications include coagulopathy, GB compactly packed with calculi, and perforated GB. ${ }^{23}$

- Equipment and technique: Percutaneous access to the GB is achieved using two methods: either transhepatic or transperitoneal route. The transhepatic route is preferred because of fewer chances of bile leak as compared with the latter. Catheter placement can be done either by trocar technique or Seldinger technique. Seldinger technique is preferred when using the transhepatic route. Under ultrasound guidance, the GB is punctured with an $18 \mathrm{G}$ percutaneous entry needle ideally at the junction of upper and middle third of body and sample aspirated. Subsequently a J-tip (Amplatz super stiff wire) guidewire is advanced through the needle and coiled into the GB. After dilation of the tract, an $8 \mathrm{~F}$ pigtail catheter is placed and connected to bag for gravity drainage ( - Fig. 2). A 10F pigtail catheter may be required to facilitate drainage in case of thick biliary secretions.

- Outcome and complication: Technical success rate is quite high, around 95 to $100 \%$. The clinical success rate varies from 60 to $90 \%$, owing to variation in the underlying clinical status of patients. The most common complication after PCC is a biliary leak. Other complications include hemobilia, hemothorax, bowel perforation, and fistula formation. The reported rate of major complication is less than $3 \% .{ }^{24-26}$ If biliary leak into the peritoneal cavity is observed while performing the procedure, the GB must be decompressed through the established access as quickly as possible to prevent biliary peritonitis. Biliary leaks secondary to catheter dislodgement should be managed by reestablishing GB drainage with a new access with additional aspiration of or catheter insertion into the leaked pericholecystic or peritoneal biliary collection.

\section{Percutaneous Transhepatic Biliary Drainage}

It is a therapeutic procedure for biliary drainage involving insertion of a biliary catheter by puncturing a peripheral intrahepatic biliary duct under image guidance. Preprocedural 
evaluation of patients involves clinical evaluation and laboratory tests, including hemogram, coagulation profile, and liver function tests. Also, cross-sectional preprocedural imaging studies should be carefully evaluated to look for the level and cause of biliary obstruction, identification for variant biliary tree anatomy, the presence of ascites, and parenchymal and portal vein status. ${ }^{27}$ If the lesion causes extrahepatic obstruction, either right or left approach may be taken. In cases in which hilum is involved, the approach should be based on liver volume status and portal vein patency. At least $30 \%$ of liver volume should be drained by a single catheter, as placement of multiple catheters is not desirable. ${ }^{1}$

\section{Procedure}

The preliminary step for PTBD is PTC. For right-sided biliary drainages, a low intercostal approach (below the 10th rib) is preferred near the midaxillary line to avoid pleural transgression. A 22G Chiba needle (part of Neff percutaneous access set; Cook) is used to puncture the dilated bile ducts under ultrasound guidance. After opacification of biliary system with small amount of contrast, a 0.018 -in nitinol guidewire is advanced through the needle into the biliary duct, over which a coaxial introducer set (part of Neff percutaneous access set; Cook) is inserted. After removing its inner components, a 0.035 -in hydrophilic guidewire is inserted into the bile duct, which is then exchanged for a stiff wire (Amplatz super stiff wire) using a 5F Kumpe catheter (Cook). Thereafter, either an internal-external biliary drainage catheter (8.3F ring biliary duct drainage catheter; Cook) or an external biliary drainage catheter (Malecot $8 \mathrm{~F}$ ) is inserted over the stiff wire after dilation of the tract.

While using fluoroscopy to puncture the bile ducts, a 22G Chiba needle is advanced from the midaxillary line directed toward the contralateral shoulder. The stylet is then removed, and the needle is slowly withdrawn while injecting small amounts of contrast. Care should be taken not to inject a lot of contrast as overdistention of biliary system can flare up sepsis. ${ }^{28}$ As the needle is slowly withdrawn, various structures (arteries and veins) may be opacified, and it is important to recognize bile ducts by their nonpulsatile slow gravitational filling. When contrast filling of bile duct occurs, site of puncture is assessed. If it is too central (first- or second-order biliary ducts), another puncture is made to target a peripheral duct (third order or more). After obtaining access to a suitable duct, a 0.018 -in nitinol guidewire is passed through the needle over which a coaxial introducer set (Neff set; Cook) is placed. After removing its inner components, a 0.035-in hydrophilic guidewire is inserted into the bile duct followed by insertion of an internal-external/external drainage catheter over a stiff wire using the same steps as described previously. For left PTBD, ultrasound guidance is invariably preferred with a subxiphoid approach, and the needle is advanced 30 to 45 degrees postero-superiorly. The commonly used hardware in PTBD is shown in - Fig. $\mathbf{3}$.

\section{Placement of an Internal-External Drain}

An internal-external biliary drainage is preferred over an external biliary drainage if the biliary obstruction can be negotiated. Internalization of the biliary drainage and placement of an internal-external biliary catheter should be attempted whenever possible, while keeping catheter-wire manipulation and contrast injection to minimum. Internal-external drainage catheter has a locking loop and multiple side holes. The catheter is positioned such that its locking loop is in the bowel, and the proximal side holes are positioned above the level of obstruction.

An external biliary drainage catheter is left in place, if the biliary obstruction cannot be negotiated. Internalization should be reattempted in such cases after 3 to 7 days of PTBD, once the biliary dilation and edema have decreased. ${ }^{29}$

The advantages of an internal-external biliary drainage over an external-only biliary drainage are that the catheters

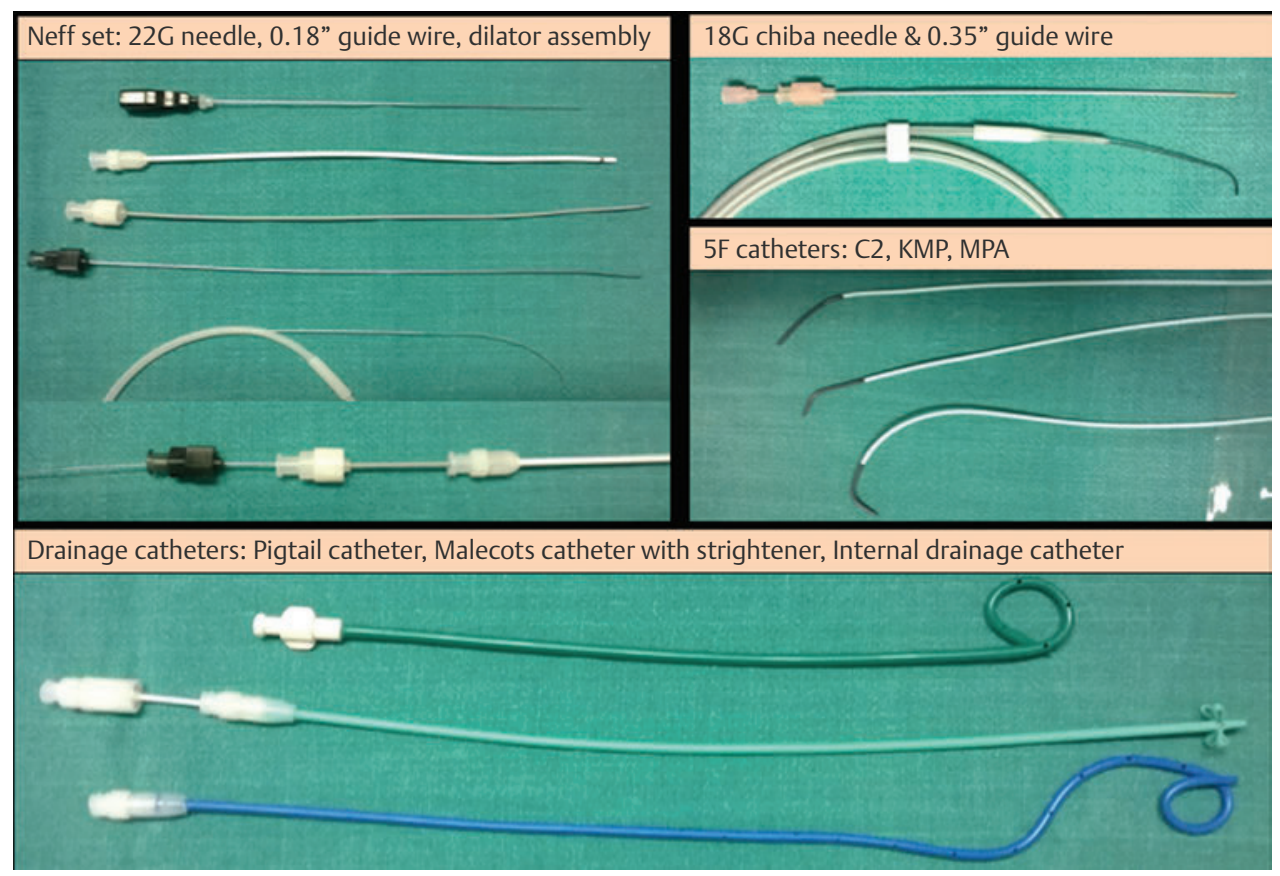

Fig. 3 Commonly used hardware in PTBD. 
are more stable and less likely to dislodge. This type of drainage is more physiologic as the bile salts, electrolytes, and fluid are not lost. The quality of life is improved as the patient can ambulate better without a bag connected to the catheter, and further excoriation of the skin around the catheter is less common as bile drains internally with reduced risk of pericatheter leak. ${ }^{29} \mathrm{After}$ the placement of an internal-external catheter, initially, the catheter is connected to an external drainage system. It can be capped if the bile is free of blood/ debris and there are no signs of cholangitis.

\section{Postprocedure Management and Catheter Care}

Correct catheter fixation to the skin is important, particularly for the right-sided biliary drainage catheters as liver movement during respiration can dislodge the catheter. If the catheter is secured tightly to the skin, there are increased chances of catheter migration as it has fixed points. Approximately $2 \mathrm{~cm}$ of slack is allowed when suturing the catheter to the skin to avoid pain and laceration at the skin site.

Postprocedure, the vital signs of the patient should be monitored for 12 to 24 hours and then as needed. The patient is administered IV antibiotics as per institution protocol (third-generation cephalosporins or piperacillin-tazobactam combination) and is maintained on IV fluids for 2 to 3 days to counter the choleresis. Laboratory investigations that indicate adequate biliary drainage and clinical improvement are total leucocyte count and liver function tests; hence, these investigations are performed daily for approximately 3 days until the pattern normalizes or stabilizes. The patient or caregiver should be educated at the time of discharge on catheter care, biliary color/output monitoring in case of external biliary drains, and on seeking immediate medical care if there is fever, abdominal/chest pain, or bloody drainage from the catheter. Regular (monthly) hospital follow-up includes monitoring the bile output and bile color along with the sonographic examination. The catheter may be flushed with $10-\mathrm{mL}$ saline under aseptic precautions, if free flow of bile is not seen. The percutaneous puncture site should be inspected for skin changes or pericatheter leakage. Internalexternal biliary drainage catheters are usually exchanged every 2 to 3 months or earlier if jaundice or cholangitis recurs.

\section{Outcome and Complications}

Technical success rate for PTBD is $100 \%$ in dilated biliary system, and in the setting of nondilated biliary ducts, $70 \%$ success rate can be anticipated as per the Society of Interventional Radiology Quality Improvement guidelines. ${ }^{30}$ The clinical outcome of biliary drainage varies widely depending on indication of PTBD. The major complications include hemorrhage, cholangitis/sepsis, pleural transgression, and death, among which hemorrhage and cholangitis/sepsis are most common with reported rate of $2.5 \%$. Other catheter-related complications include pericatheter biliary leakage and absence or fall in the biliary output.

\section{Management of Complications}

The most common complication following PTBD is the perior intracatheter hemorrhage that occurs usually due to the abnormal retracted catheter location, where the proximal side hole of the catheter is in the hepatic parenchymal tract. This can be easily detected and managed by advancing the catheter under fluoroscopic guidance, such that the proximal side hole of the catheter is within the bile duct. If bleeding persists and the patient is hemodynamically stable, the catheter can be upsized by $2 \mathrm{~F}$ ( $8 \mathrm{~F}$ catheter upsized to $10 \mathrm{~F}$ catheter). Emergent angiogram and embolization must be performed if the patient is hemodynamically unstable after fluid resuscitation or if bleeding persists despite upsizing the catheter as the source of bleed may be an injured hepatic artery. ${ }^{31}$

Cholangitis following PTBD is managed by IV antiobiotic course and connecting the internal-external biliary drainage catheter to the bag to facilitate the drainage of infected bile. ${ }^{32}$ The catheter-related complications such as pericatheter biliary leakage can be managed by repositioning the catheter or upsizing the biliary catheter by $2 \mathrm{~F}$ as mentioned previously. Absent or decreased biliary output may be related to catheter blockage, dislodgement, or kinking. A blocked catheter can be managed by flushing or replacing the catheter. Partially dislodged or kinked catheter may be repositioned/reinserted after manipulation; however, a new PTBD is the only option if the catheter is completely dislodged from the liver surface and the old PTBD tract is not mature. ${ }^{31}$

\section{Percutaneous Transhepatic Biliary Drainage in Malignant Biliary Tract Obstruction}

Percutaneous transhepatic biliary drainage is required in malignant biliary tract obstruction for the relief of pruritus and cholangitis. Apart from that, PTBD is indicated to reduce serum bilirubin to facilitate chemotherapy and prior to surgery. PTBD is also done in association with endoluminal brush biopsy, particularly for the lesions that are not amenable to biopsy/fine-needle aspiration biopsy (FNAC) through percutaneous route. ${ }^{27}$ For surgically unresectable patients, PTBD is the first step for palliative procedures such as the placement of biliary stents, intraluminal brachytherapy (ILBT), endobiliary radiofrequency ablation, and photodynamic therapy (PDT).

Before planning PTBD in malignancies, pathologic anatomy should always be assessed as malignancies tend to separate one part of the biliary system from the other, which is termed as isolation. Bismuth-Corlette classification of hilar cholangiocarcinoma ( - Figs. $\mathbf{4}, \mathbf{5}$ ) provides a useful scheme for describing high bile duct obstructions. Type 1 pattern spares the primary confluence, so this can be treated with a single catheter or stent from either a right- or left-sided approach. Type 2 obstructions involve primary confluence; therefore, complete drainage of such obstructions requires placement of bilateral internal-external biliary drainage catheters. In type 3 obstructions, complete drainage of either the right (type $3 \mathrm{~A}$ ) or the left (type $3 \mathrm{~B}$ ) requires additional drains or stents. Type 4 obstructions involve bilateral secondary confluences. For patients with such type of obstruction, the appropriateness of biliary drainage should be reconsidered on a case-by-case basis in view of the indication, chances of success, and attendant complications. ${ }^{11}$ 

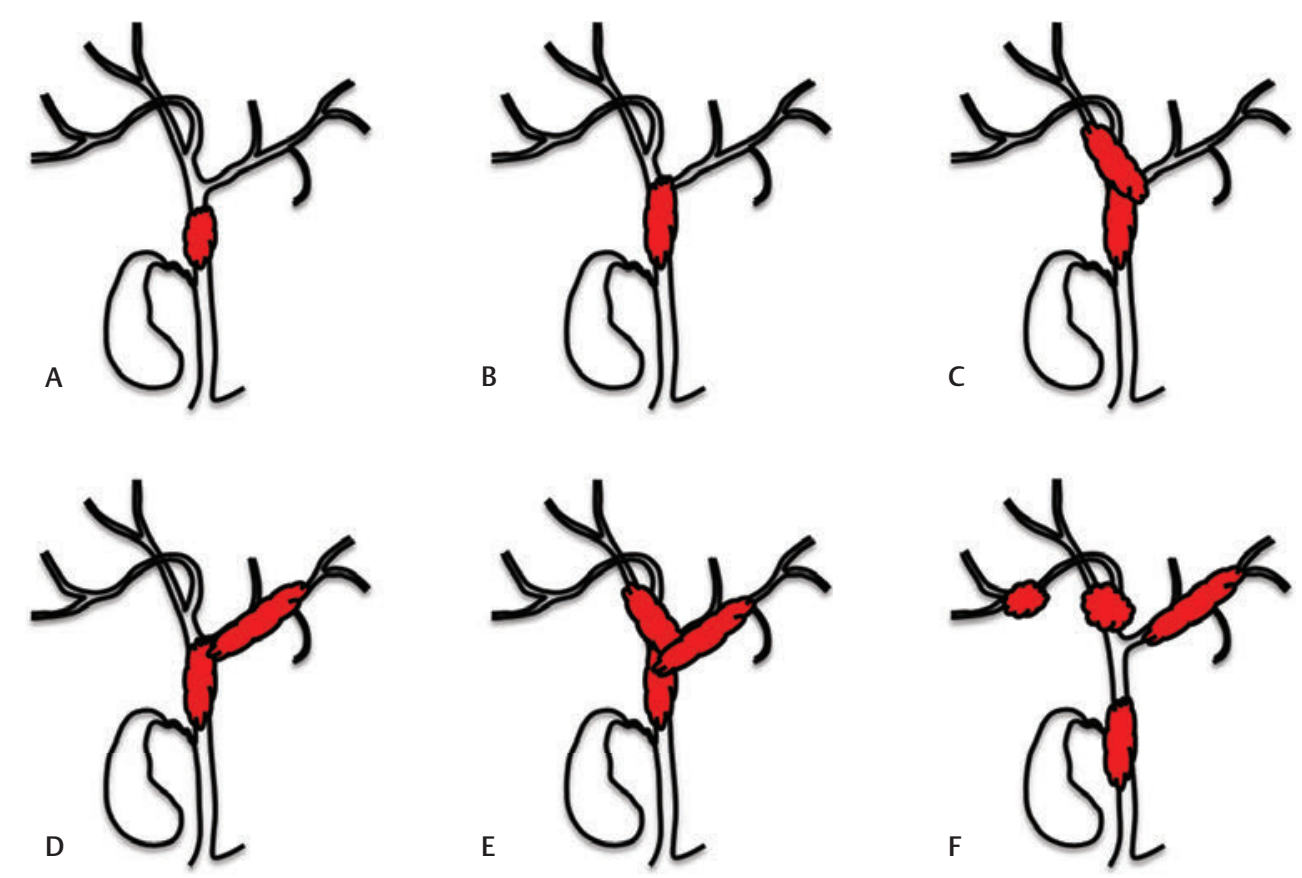

Fig. 4 Diagrammatic representation of Bismuth-Corlette classification for perihilar cholangiocarcinoma.
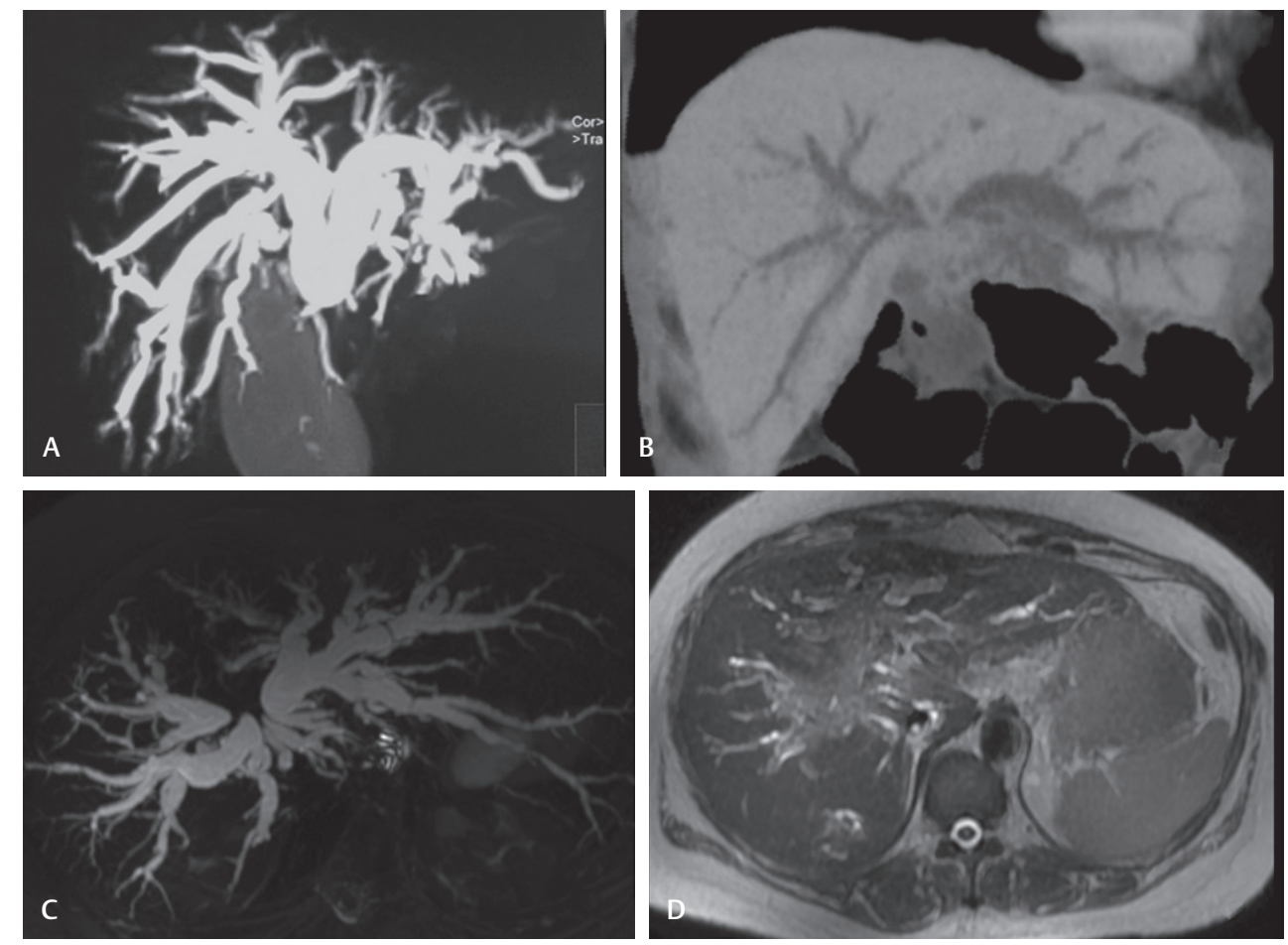

Fig. 5 Bismuth-Corlette classification. (A) Type 1 block. (B) Type 2 block. (C) Type 3 block (involvement of right secondary confluence). (D) Type 4 block.

\section{Biliary Stenting}

Biliary stents can be made of either plastic or metal. Metallic stents are only used in malignant obstructions in nonsurgical and noncholangitic patients. Metallic biliary stents are inserted in contracted state over the wire through PTBD tract, and then they achieve large lumen following expansion. They are expensive but are less prone to migration and occlusion as compared with plastic stents. Balloon dilation of malignant biliary tract obstructions prior to stent placement should not be done as this can cause hemobilia. ${ }^{31}$ For lesions causing isolation of right and left ducts, placement of metallic stents in either a T ( - Fig. 6) or Y ( - Fig. 7) configuration can be done, and in high biliary tract obstructions, hilar reconstruction can also be done with the help of stents ( - Fig. 8). Median stent patency is around 6 to 9 months. ${ }^{11}$ Around 10 to $30 \%$ patients may require reintervention for occlusion of metallic 


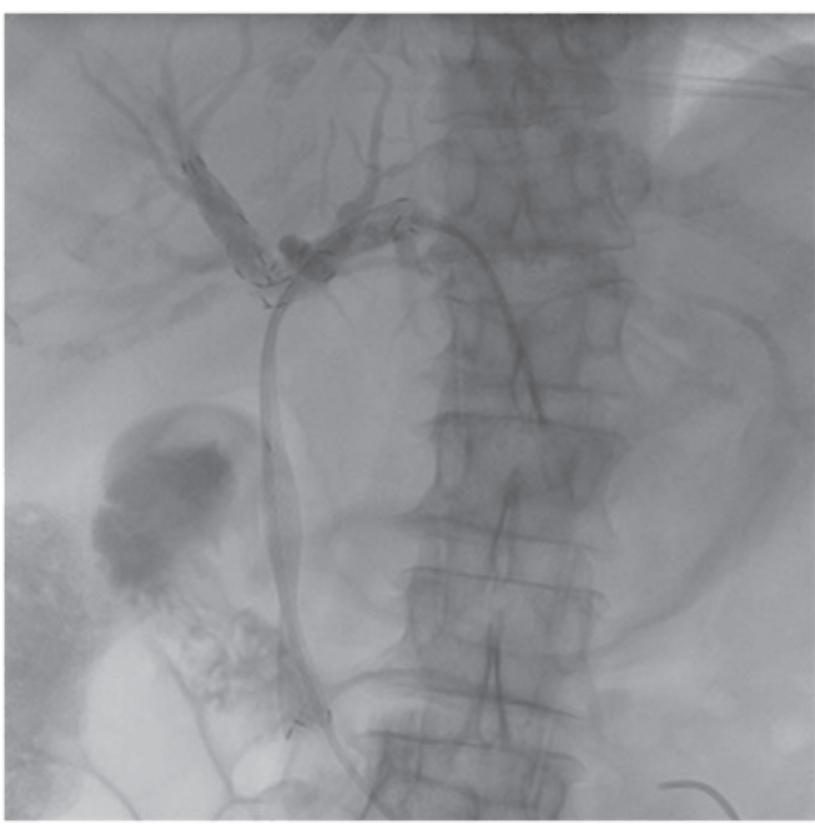

Fig. 6 T-type stenting: Two metal stents placed in T configuration.

biliary stents. Use of polytetrafluoroethylene (PTFE)-covered stents has also been evaluated to prolong stent patency by minimizing tumor in growth. However, there is higher chance of occlusion of side branches, cystic duct, and main pancreatic duct (MPD) as compared with bare metal stents. ${ }^{33}$

The most common complications with biliary stenting include pancreatitis, cholangitis, and stent occlusion. Stent occlusion may occur due to tumor in-growth/overgrowth, debris, clots, or stones. Stent occlusion can be managed by performing a new PTBD followed by recanalization of the stent or placing a new stent within the preexisting stent

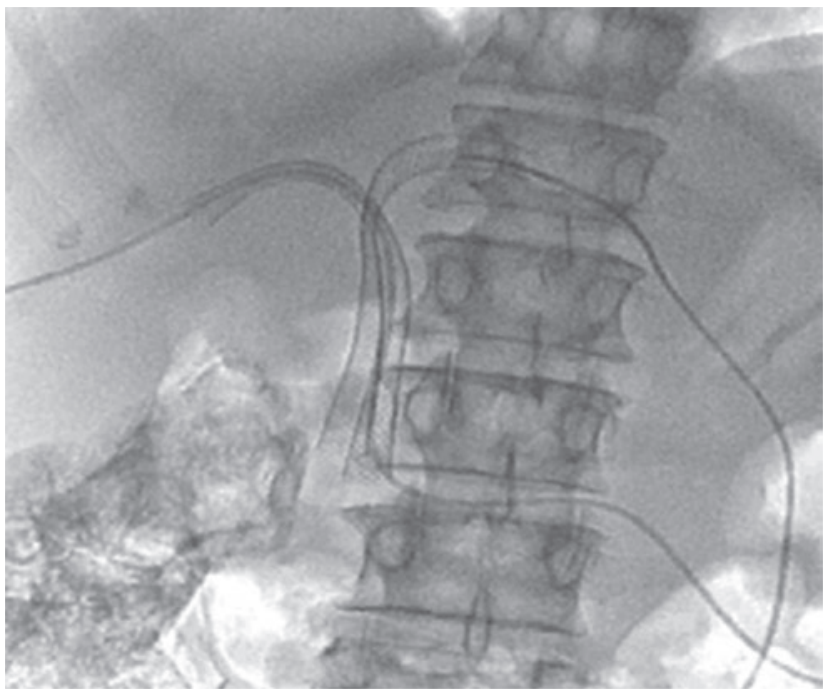

Fig. 7 Y-type stenting: Two metal stents placed in Y configuration.

or an internal external biliary drainage catheter within the stent. Pancreatitis secondary to biliary stenting is managed medically by aggressive hydration, maintaining electrolyte balance and preventing associated complications. Prolonged antiobiotic course is required in cases complicated by cholangitis, and a new PTBD with external biliary drainage is performed if the cholangitis is secondary to stent occlusion. Stent recanalization, as mentioned previously, is performed once the cholangitis has resolved.

\section{Intraluminal Brachytherapy}

High-dose ILBT using iridium 192 can be delivered selectively to the tumor avoiding the side effects of external beam
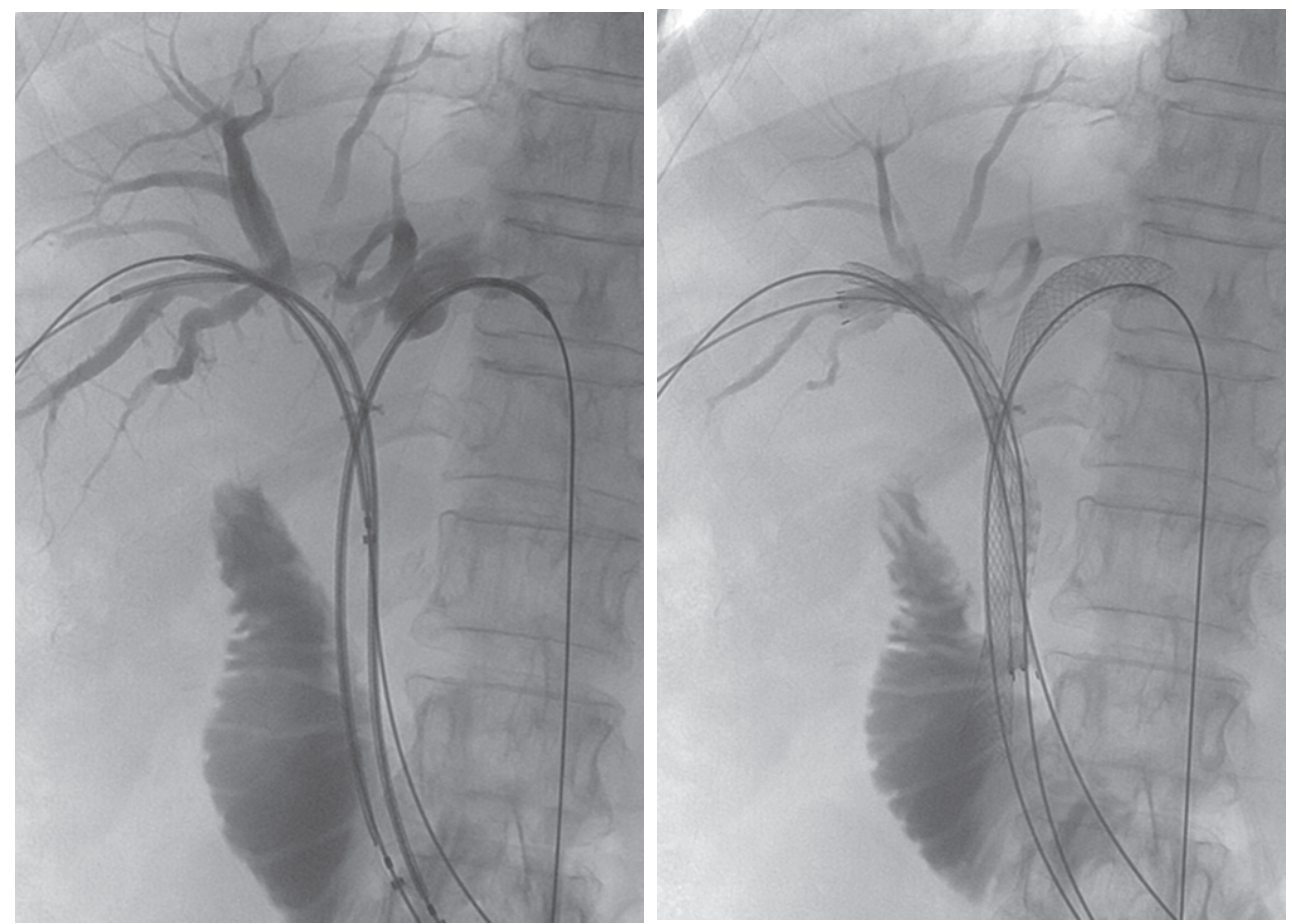

Fig. 8 Three self-expandable metal stents placed in high biliary duct obstruction (Type 3a block). 
radiotherapy. Studies have shown that PTBD followed by ILBT is a feasible procedure with good symptom control, definite impact on quality of life, and minimal complications. ${ }^{34}$ It has also been used post-stenting and documented to prolong stent patency. In a study conducted by Chen et al, the authors showed that the mean stent patency was significantly longer in the group in which ILBT was given (12.6 months) than in the control group (8.3 months) in which ILBT was not given. ${ }^{35}$

\section{Photodynamic Therapy for Bile Duct Cancer}

Photodynamic therapy is a local photochemical tumor treatment that consists of a photosensitizing agent (Porfimer) in combination with laser irradiation of a distinct wavelength. Tumor ablation with PDT combined with biliary stenting reduces cholestasis and significantly improves median survival time and quality of life in selected patients with bile duct cancers. However, it cannot eradicate the primary tumor when tumor invasion extends to a depth of 7 to $9 \mathrm{~mm}$. Therefore, the appropriate indications of PDT may be as follows: (1) sclerosing variant without hematogenous metastasis; (2) superficial spreading type with the papillary variant; and (3) R0 and R1 residual tumor after resection. PDT has shown its efficacy in improving survival, jaundice, and quality of life in unresectable cases. ${ }^{36}$ Also, PDT was evaluated as a neoadjuvant therapy in advanced malignancies, and extensive resections can be performed in combination with PDT, which allows the inclusion of patients who were once deemed unresectable. ${ }^{37}$

\section{Endobiliary Radiofrequency Ablation}

Endobiliary radiofrequency ablation (-Fig. 9) has been shown to be an effective palliative modality in the treatment of malignant biliary obstruction by delaying tumor growth and increasing the duration of stent patency. ${ }^{38}$

Successful use of endobiliary radiofrequency ablation combined with balloon sweep technique to establish patency of blocked metallic biliary stents has been reported previously ${ }^{39,40}$ This approach can clear the occlusion and restore biliary flow without the need to insert a new stent within the obstructed stent, thereby saving the cost of a second stent. Reopened stent also offers the medical oncologist a chance of administering additional chemotherapy that improves patient's survival. ${ }^{39,40}$

\section{Percutaneous Transhepatic Biliary Drainage in Benign Biliary Tract Obstruction}

Benign biliary strictures are either (1) peripheral intrahepatic and/or (2) central, involving common hepatic duct (CHD), left hepatic duct (LHD), or right hepatic duct (RHD) (anastomotic/nonanastomotic) biliary strictures. In cases of peripheral stricture, if the patient is asymptomatic and has no cholangitis, intervention is not needed as there is eventual atrophy of the involved hepatic segment, with compensatory hypertrophy of adjacent hepatic segments. The only exception is an undersized hepatic graft (usually a pediatric recipient) with the involvement of a relatively large hepatic segment. ${ }^{32,41}$ In central benign strictures, indications for PTBD include cholangitis, biliary stones, cholestasis with pruritus, abnormal liver function tests, and hepatic graft dysfunction.

Balloon dilation of these strictures with high-pressure low compliance balloons following PTBD is an effective therapeutic option. The technique consists of crossing the stricture with a hydrophilic guidewire (Terumo, Japan) after performing a PTBD. The stricture is inflated with an 8- to $10-\mathrm{mm}$ balloon, and an internal external catheter (10-12F) is placed across the stricture for 3 to 4 weeks and capped (-Fig. 10) to induce nonstenosed fibrosis. The patient is monitored, and check cholangiogram is taken to ensure the free flow of bile into the bowel. The procedure is repeated, if needed. The external catheter is removed only if the cholangiographic findings are satisfactory, the patient remains asymptomatic, and laboratory parameters are normal. Success rates of this technique are variable with a reported failure rate of up to $45 \%$ owing to the fibrous nature of these strictures. The peripheral cutting balloons were also proven to be effective in biliary strictures not responsive to standard measures using conventional high-pressure balloons. ${ }^{42}$ Use
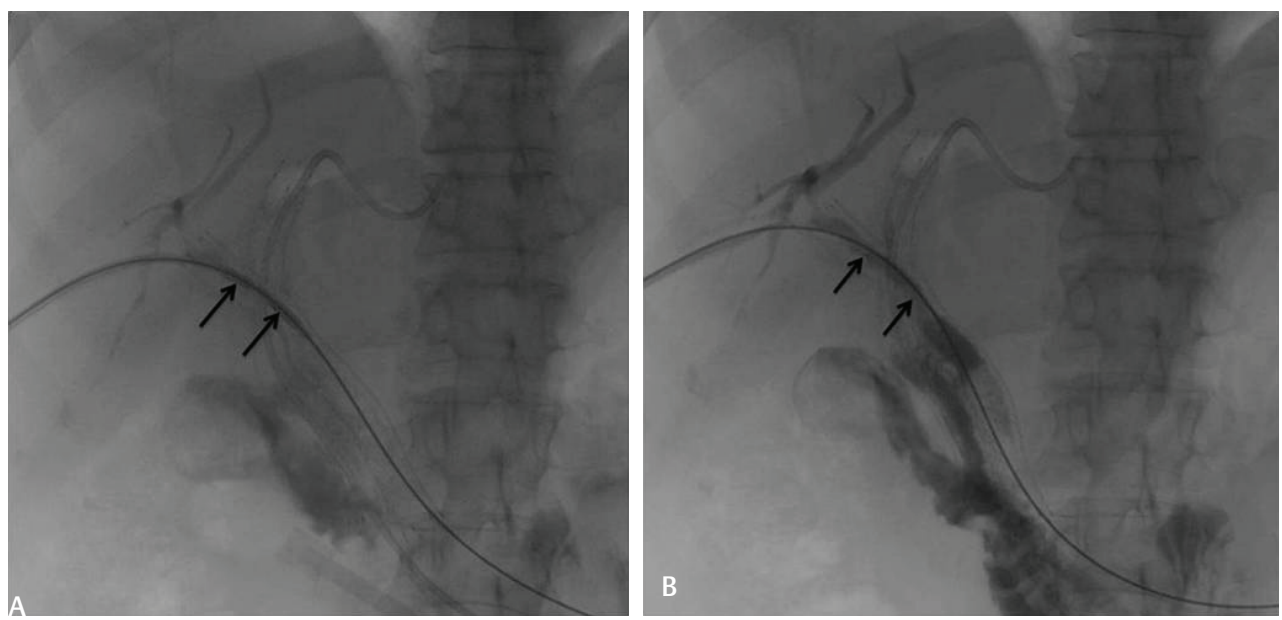

Fig. 9 Fluoroscopic images show endobiliary RF ablation (arrows showing ring electrodes) being performed (A) and reopened stent with contrast agent flow (arrows) within the stent (B). 

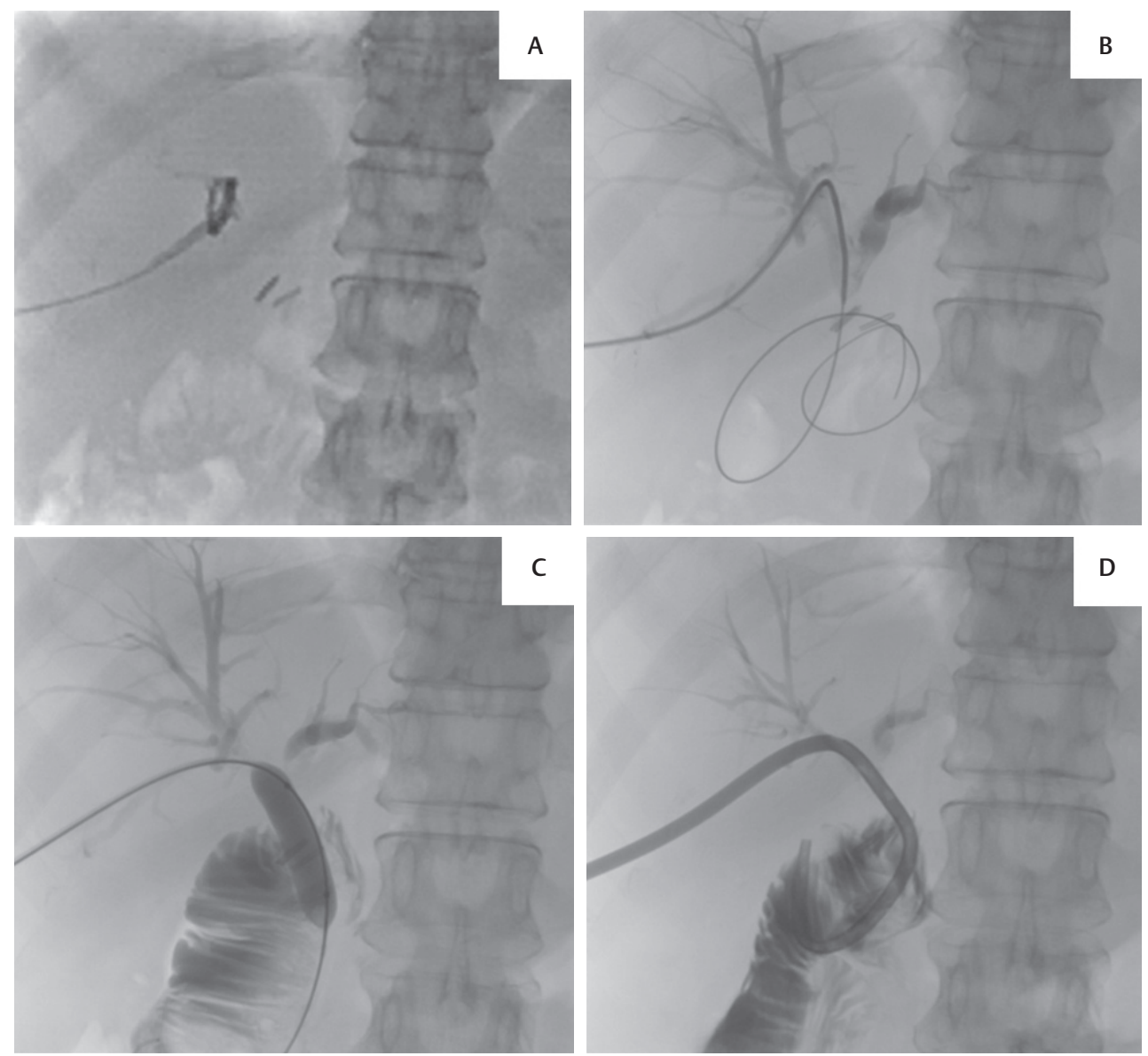

Fig. 10 Balloon dilation of post hepaticojejunostomy stricture. (A) Initial access to right-sided biliary ducts. (B) Narrowing at HJ anastomotic site with upstream biliary radical dilation and a hydrophilic guidewire is seen across the narrowing. (C) Dilation of stricture using a 10-mm high-pressure balloon. (D) Placement of internal external drainage catheter across the narrowing.

of removable fully covered metal stents with a dwell time of around 4 to 5 weeks is also effective for the treatment of benign biliary strictures. ${ }^{8}$

\section{Percutaneous Transhepatic Biliary Drainage in Biliary Leaks}

Biliary leaks can be secondary to iatrogenic injuries or related to trauma. Small leaks can be managed conservatively with antibiotics and placement of percutaneous drains, but massive biliary leaks can result in bilomas, fistulas, and biliary peritonitis, with subsequent sepsis. PTBD in such cases is needed when definitive healing of the injured bile duct is expected after endoluminal decompression. PTBD serves to decompress the biliary system and redirect bile flow from the defect into the bile ducts. This may lead to sufficient healing of the site of leakage, and additional surgery may not be necessary. In critically ill patients, it may allow the patient's condition to improve before subsequent definitive surgery. Because of the more invasive nature of percutaneous biliary access, ERCP-mediated biliary decompression is the first choice. However, when ERCP is not feasible, PTBD is the only option. While placing an internal-external catheter, care should be taken to place side holes on both sides of the bile leak to keep intraluminal resistance as low as possible.
For biliary leakage, PTBD has been reported to be effective in 50 to $100 \%$ of patients without additional surgery. ${ }^{43}$ Also, retrievable covered stents have been used effectively for treating postoperative bile leak as well as combined biliary stricture. ${ }^{44}$ The liquid embolic agents (NBCA [ $N$-butyl cyanoacrylate] glue) have also been used to achieve therapeutic success in biliary leaks by causing obliteration of isolated segmental biliary ducts responsible for persistent biliary leaks. ${ }^{45,46}$

\section{Percutaneous Transhepatic Biliary Drainage in the Management of Biliary Calculi}

The principal indication for gallstone extraction through percutaneous route is the treatment of symptomatic calculi in patients who are not candidates for surgery. Apart from that, patients with retained biliary duct stones following surgery will also be referred for percutaneous management following failed ERCP. Peripheral to the hilum of the liver, intraductal calculi are beyond the reach of endoscopists, and percutaneous management plays a larger role. The procedure is generally staged so that initially the patients will undergo drainage of the GB or placement of a PTBD catheter. This is followed by tract dilation and stone removal, and later tract evaluation and tube removal. GB stones are usually extracted through large sheaths using graspers and baskets. Stones too large to 
remove via the sheath and resistant to basket fragmentation can be broken up with the EHL (intracorporeal electrohydraulic lithotripsy) probe.

Two methods to remove ductal calculi are either balloon dilation of the ampulla followed by use of balloons to dislodge and push calculi into the bowel (this technique works best for smaller, nonimpacted stones) or by the use of basket/ EHL fragmentation that is necessary to treat larger calculi. ${ }^{47}$

\section{Conclusion}

Percutaneous transhepatic biliary interventions have become the mainstay in the management of hepato biliary diseases not amenable to ERCP. The fact that they are less invasive and safer has made them the most sought after. The principal procedures include percutaneous cholecystotomy (PCC) and PTBD. PCC, a minimally invasive procedure in acute cholecystitis, is a bridge to definitive surgery and may be the only procedure that can be offered to the critically ill and poor surgical candidates with biliary sepsis. PTBD is an indispensable therapeutic procedure for biliary drainage to relieve benign or malignant obstruction when endoscopic biliary drainage has failed or cannot be performed.

Metallic biliary stenting following PTBD is the best palliation, and it improves the quality of life in patients with inoperable malignant biliary obstruction. Endobiliary radiofrequency ablation, ILBT, and photodynamic therapy are effective palliative modalities in the management of malignant biliary obstruction.

The outcome of percutaneous transhepatic biliary interventions can be improved by in-depth knowledge of the biliary anatomy and variants as well as the technical considerations and importantly wise patient selection.

\section{Conflict of Interest}

None.

\section{References}

1 Gupta AK, Chowdhary V, Khandelwal N. Diagnostic Radiology: Gastrointestinal and Hepatobiliary Imaging. 3rd ed. New Delhi, India: Jaypee Brothers; 2009:394-402

2 Ring EJ, Kerlan RK Jr. Interventional biliary radiology. AJR Am J Roentgenol 1984;142(1):31-34

3 McLean GK, Ring EJ, Freiman DB. Therapeutic alternatives in the treatment of intrahepatic biliary obstruction. Radiology 1982;145(2):289-295

4 Crummy AB, Turnipseed WD. Percutaneous replacement of a biliary T tube. AJR Am J Roentgenol 1977;128(5):869-870

5 Pomerantz BJ. Biliary tract interventions. Tech Vasc Interv Radiol 2009;12(2):162-170

6 Lipsett PA, Pitt HA. Cholangitis. In: Blumgart L, Belghiti J, Jarnagin WR, et al eds. Surgery of the Liver, Biliary Tract and Pancreas, vol 2. 4th ed. Philadelphia, PA: Saunders; 2007:917-926

7 Costamagna G, Boškoski I. Current treatment of benign biliary strictures. Ann Gastroenterol 2013;26(1):37-40

8 Gwon DI, Laasch HU. Radiological approach to benign biliary strictures. Gastrointestinal Intervention 2015;4:9-14

9 Dadhwal US, Kumar V. Benign bile duct strictures. Med J Armed Forces India 2012;68(3):299-303
10 Cannavale A, Santoni M, Gazzetti M, et al. Updated management of malignant biliary tract tumors: an illustrative review. J Vasc Interv Radiol 2016;27(7):1056-1069

11 Thornton RH, Covey AM. Management of malignant biliary tract obstruction. In: Mauro MA, Murphy KPJ, Thomson KR, Venbrux AC, Morgan RA, eds. Image-Guided Interventions. 2nd ed. Philadelphia, PA: Saunders;2014:981-993

12 Madhusudhan KS, Gamanagatti S, Gupta AK. Imaging and interventions in hilar cholangiocarcinoma: a review. World J Radiol 2015;7(2):28-44

13 Sureka B, Bansal K, Patidar Y, Arora A. Magnetic resonance cholangiographic evaluation of intrahepatic and extrahepatic bile duct variations. Indian J Radiol Imaging 2016;26(1):22-32

14 Covey AM, Brown KT. Percutaneous transhepatic biliary drainage. Tech Vasc Interv Radiol 2008;11(1):14-20

15 Moon E, Tam MDBS, Kikano RN, Karuppasamy K. Prophylactic antibiotic guidelines in modern interventional radiology practice. Semin Intervent Radiol 2010;27(4):327-337

16 Tuite C, Rosenberg EJ. Sedation and analgesia in interventional radiology. Semin Intervent Radiol 2005;22(2):114-120

17 Hatzidakis AA, Charonitakis E, Athanasiou A, et al. Sedations and analgesia in patients undergoing percutaneous transhepatic biliary drainage. Clin Radiol 2003;58(2):121-127

18 Garg R, Pandey R, Darlong V, Punj J. Anaesthetic considerations for Interventional Radiology. Int J Anesthesiol 2008;19 (1):87-94

19 Atkinson M, Happey MG, Smiddy FG. Percutaneous transhepatic cholangiography. Gut 1960;1(4):357-365

20 Macrì A, Scuderi G, Saladino E, et al. Acute gallstone cholecystitis in the elderly: treatment with emergency ultrasonographic percutaneous cholecystostomy and interval laparoscopic cholecystectomy. Surg Endosc 2006;20(1):88-91

21 Akyürek N, Salman B, Yüksel O, et al. Management of acute calculous cholecystitis in high-risk patients: percutaneous cholecystotomy followed by early laparoscopic cholecystectomy. Surg Laparosc Endosc Percutan Tech 2005;15(6):315-320

22 Picus D, Hicks ME, Darcy MD, et al. Percutaneous cholecystolithotomy: analysis of results and complications in 58 consecutive patients. Radiology 1992;183(3):779-784

23 Fahrbach TM, Wyse GM, Lawler LP, Kim HS. Percutaneous cholecystotomy. In: Mauro MA, Murphy KPJ, Thomson KR, Venbrux AC, Morgan RA, eds. Image-Guided Interventions. 2nd ed. Philadelphia, PA: Saunders; 2014:1008-1013

24 Viste A, Jensen D, Angelsen JH, Hoem D. Percutaneous cholecystostomy in acute cholecystitis; a retrospective analysis of a large series of 104 patients. BMC Surg 2015;15:17

25 Vogelzang RL, Nemcek AA Jr. Percutaneous cholecystostomy: diagnostic and therapeutic efficacy. Radiology 1988;168 (1):29-34

26 vanSonnenberg E, D’Agostino HB, Goodacre BW, Sanchez RB, Casola G. Percutaneous gallbladder puncture and cholecystostomy: results, complications, and caveats for safety. Radiology 1992;183(1):167-170

27 Madhusudhan KS, Gamanagatti S, Srivastava DN, Gupta AK. Radiological interventions in malignant biliary obstruction. World J Radiol 2016;8(5):518-529

28 Ferrucci JT Jr, Mueller PR, Harbin WP. Percutaneous transhepatic biliary drainage: technique, results, and applications. Radiology 1980;135(1):1-13

29 Chandrashekhara SH, Gamanagatti S, Singh A, Bhatnagar S. Current status of percutaneous transhepatic biliary drainage in palliation of malignant obstructive jaundice: a review. Indian J Palliat Care 2016;22(4):378-387

30 Saad WE, Wallace MJ, Wojak JC, Kundu S, Cardella JF. Quality improvement guidelines for percutaneous transhepatic cholangiography, biliary drainage, and percutaneous cholecystostomy. J Vasc Interv Radiol 2010;21(6):789-795 
31 Das A, Baliyan V, Gamanagatti S, Gupta AK. Percutaneous biliary intervention: tips and tricks. Trop Gastroenterol 2017; 38(2):71-89

32 Cantwell CP, Pena CS, Gervais DA, Hahn PF, Dawson SL, Mueller PR. Thirty years' experience with balloon dilation of benign postoperative biliary strictures: long-term outcomes. Radiology 2008;249(3):1050-1057

33 van Delden OM, Laméris JS. Percutaneous drainage and stenting for palliation of malignant bile duct obstruction. Eur Radiol 2008;18(3):448-456

34 Aggarwal R, Patel FD, Kapoor R, Kang M, Kumar P, Chander Sharma S. Evaluation of high-dose-rate intraluminal brachytherapy by percutaneous transhepatic biliary drainage in the palliative management of malignant biliary obstruction-a pilot study. Brachytherapy 2013;12(2):162-170

35 Chen Y, Wang X-L, Yan Z-P, et al. HDR-192Ir intraluminal brachytherapy in treatment of malignant obstructive jaundice. World J Gastroenterol 2004;10(23):3506-3510

36 Lee TY, Cheon YK, Shim CS. Current status of photodynamic therapy for bile duct cancer. Clin Endosc 2013;46(1):38-44

37 Wiedmann M, Caca K, Berr F, et al. Neoadjuvant photodynamic therapy as a new approach to treating hilar cholangiocarcinoma: a phase II pilot study. Cancer 2003;97(11):2783-2790

$38 \mathrm{Hu}$ B, Gao DJ, Zhang X, Zhang YC. Endobiliary radiofrequency ablation improve overall survival of cholangiocarcinoma: a multi-center randomized control study. Gastrointest Endosc 2016;83(5):AB126

39 Mukund A, Arora A, Rajesh S, Bothra P, Patidar Y. Endobiliary radiofrequency ablation for reopening of occluded biliary stents: a promising technique. J Vasc Interv Radiol 2013;24(1):142-144

40 Betgeri S, Rajesh S, Arora A, Panda D, Bhadoria AS, Mukund A. Percutaneous endobiliary RFA combined with balloon-sweep for re-opening occluded metallic biliary stents. Minim Invasive Ther Allied Technol 2017;26(2):124-127

41 Saad WE. Management of benign biliary strictures. In: Mauro MA, Murphy KPJ, Thomson KR, Venbrux AC, Morgan RA, eds. Image-Guided Interventions. 2nd ed. Philadelphia, PA: Saunders; 2014:994-1001

42 Mukund A, Rajesh S, Agrawal N, Arora A, Arora A. Percutaneous management of resistant biliary-enteric anastomotic strictures with the use of a combined cutting and conventional balloon cholangioplasty protocol: a single-center experience. J Vasc Interv Radiol 2015;26(4):560-565

43 Portugaller RH, Hausegger KA. Management of biliary leaks. In: Mauro MA, Murphy KPJ, Thomson KR, Venbrux AC, Morgan RA, eds. Image-Guided Interventions. 2nd ed. Philadelphia, PA: Saunders; 2014:1002-1006

44 Gwon DI, Ko G-Y, Sung K-B, Kim J-H, Yoon H-K. Percutaneous transhepatic treatment of postoperative bile leaks: prospective evaluation of retrievable covered stent. J Vasc Interv Radiol 2011;22(1):75-83

45 Zarghouni M, Cura M, Kim PT, Testa G, Klintmalm GB. Segmental biliary duct $\mathrm{N}$-butyl cyanoacrylate ablation in a transplant liver for a biliary leak after hepaticojejunostomy.-butyl cyanoacrylate ablation in a transplant liver for a biliary leak after hepaticojejunostomy. Liver Transpl 2014;20(6):739-741

46 Vu DN, Strub WM, Nguyen PM. Biliary duct ablation with $N$-butyl cyanoacrylate. J Vasc Interv Radiol 2006;17(1):63-69

47 Brown DB, Picus DD. Management of biliary calculi. In: Mauro MA, Murphy KPJ, Thomson KR, Venbrux AC, Morgan RA, eds. Image-Guided Interventions. 2nd ed. Philadelphia, PA: Saunders; 2014:1014-1019 